\title{
Harmonic scalpel harvest of bilateral internal thoracic arteries
}

\author{
Teresa M. Kieser \\ Department of Cardiac Sciences, Libin Cardiovascular Institute of Alberta, University of Calgary, Calgary, Alberta, Canada \\ Correspondence to: Teresa M. Kieser. Department of Cardiac Sciences, Libin Cardiovascular Institute of Alberta, University of Calgary, Rm C814, \\ Foothills Medical Centre, 1403 29th St NW, Calgary, Alberta T2N 2T9, Canada. Email: t.kieserprieur@ucalgary.ca.
}

Submitted Mar 20, 2018. Accepted for publication May 28, 2018.

doi: $10.21037 /$ acs.2018.06.02

View this article at: http://dx.doi.org/10.21037/acs.2018.06.02

\section{Clinical vignette}

A 57-year-old male with end-stage renal failure was discovered to have double vessel coronary disease precluding his chance for renal transplant. He underwent a double coronary artery bypass graft (CABG) off-pump surgery with two in situ internal thoracic arteries (ITA) to the left anterior descending artery (LAD) (with 70\% stenosis) and right posterior descending artery (PDA), (with $90 \%$ stenosis of right main coronary artery).

\section{Surgical techniques}

\section{Preparation}

The pericardium is opened and the coronary targets are assessed for graftability. Test the harmonic scalpel (HS) (HARMONIC SYNERGY Hook Blade Ethicon Endo Surgery, CVD, Minneapolis, MN, USA) and position the hook with the blunt aspect as the primary dissecting edge.

\section{Exposition}

The ITA retractor of choice is inserted and the visceral pleura is swept laterally with gentle blunt and cautery dissection to keep the pleura intact if possible to a point 1-2 $\mathrm{cm}$ lateral to the ITA and veins. Drawing a line from where the ITA is seen (usually in the mid-section of the operative site) caudally, incise with Metzenbaum scissors at the most caudal point in a crevice between the medial IT vein and ITA to expose the length of the ITA.

\section{Operation}

Dissection of the ITA with the HS can be divided into three sections: the start-up at the caudal end, the middle segment and the proximal segment at the cephalad end. The HS is used to 'paint' the ITA with light strokes briefly touching the artery sweeping the peri-mammary tissues away. Branches of the ITA, usually at the intercostal interspaces are sealed with the blunt end of the HS placed at $1 \mathrm{~mm}$ away from the ITA with the help of forceps placed with one prong either side of the branch depressing the ITA (so as to avoid damage to the ITA). The ITA is circumferentially harvested from caudal to cephalad end enabling proximal and distal control should repair with suture of an avulsed side branch be necessary.

\section{Completion}

The end of the dissection occurs when the first intercostal branch at the upper end is sealed; one knows this by feeling no restrictions or tethering of the ITA when passing the index finger along the ITA above this sealed branch toward the subclavian artery.

\section{Comments}

\section{Clinical results}

Use of the HS for ITA harvest began in the late 1990s for minimally invasive CABG surgery (1). In a retrospective review of 2,205 ITA grafts from 1,787 ITA conduits in 1,051 consecutive patients, patients with skeletonization with use of the HS ( $\mathrm{N}=965)$ were compared to those without use of the HS $(\mathrm{N}=86)$ (2). Results were similar with respect to reoperation for bleeding, (2.7\% vs. $3.5 \%, \mathrm{P}=0.726)$, early reoperation for failed grafts $(0.7 \%$ vs. $0, \mathrm{P}=1.0)$, deep sternal infection $(1.6 \%$ vs. $1.2 \%, \mathrm{P}=1.0)$, post-operative myocardial infarction $(1.7 \%$ vs. $2.3 \%, \mathrm{P}=0.653)$ and total operative 
mortality ( $3.7 \%$ vs. $2.3 \%, \mathrm{P}=0.763)$. Use of the HS facilitated skeletonization of the ITA by being faster: non HS skeletonization harvest time of 77 ITAs averaged 32.2 minutes compared to times for the last 100 HS skeletonized ITAs of the study of 15.4 minutes. Damage to the ITA by the HS was also infrequent: 7 of 1,640 (0.43\%) were not usable. Other authors have found that use of the HS not only decreases operative time but also conduit spasm and reduces the number of clips (3).

\section{Advantages}

Benefits of HS use include less conduit spasm, damage and use of clips. Fewer clips facilitate sequential ITA grafting and the ITA does not have 'indents' at clipped branch locations when the ITA spasm releases, even if one placed clips at a thought-to-be far enough distance from the main body of the ITA. The faster harvest time is important for allsurgeons, patients and the operative team. If harvest time is reduced by 15 minutes/ITA, in a 2 CABG day with use of bilateral ITA in both operations, the day could potentially be finished at 17:00 hours instead of 18:00 hours.

\section{Caveats}

The main disadvantage of the HS is its inability to 'cauterize' bleeding vessels. For chest wall bleeding, cautery is used but for ITA bleeding either clips or suture with 7.0 or 8.0 Prolene must be used. It is also difficult to 'seal' branches in patients on intravenous heparin, or P2Y12 inhibitors such as Clopidogrel or P2Y12 antagonists such as Ticagrelor. Patients with long-standing diabetes mellitus may have fibrosed peri-thoracic artery tissues making harvest longer and more tedious. The learning curve for use of the HS is approximately 20 harvests if a surgeon is not experienced with the skeletonization technique and 10 harvests if

Cite this article as: Kieser TM. Harmonic scalpel harvest of bilateral internal thoracic arteries. Ann Cardiothorac Surg 2018;7(5):698-699. doi: 10.21037/acs.2018.06.02 experienced.

\section{Summary}

Use of harmonic technology facilitates use of ITAs for arterial CABG by being faster, less damaging, with fewer use of clips and causing less ITA spasm. With increased ease of harvest of arterial conduits, use of bilateral ITA grafting may increase, which will benefit patients long-term.

\section{Acknowledgements}

The author wishes to thank Ethicon Endosurgery for their support of education on the Harmonic Scalpel by funding the first movie by this author 'HYPERLINK' /article/ skeletonization-internal-mammary-artery-harmonic-hookblade 'Skeletonization of the Internal Mammary Artery with the Harmonic Hook Blade' published on CTSNet July 12, 2012.

\section{Footnote}

Conflicts of Interest: The author has no conflicts of interest to declare.

\section{References}

1. Wolf RK, Ohtsuka T, Flege JB Jr. Early results of thoracoscopic internal mammary artery harvest using an ultrasonic scalpel. Eur J Cardiothorac Surg 1998;14 Suppl 1:S54-7.

2. Kieser TM, Rose MS, Aluthman U, et al. Quicker yet safe: skeletonization of 1640 internal mammary arteries with harmonic technology in 965 patients. Eur J Cardiothorac Surg 2014;45:e142-50.

3. Isomura $\mathrm{T}$, Suma H, Sato T, et al. Use of the Harmonic Scalpel for harvesting arterial conduits in coronary artery bypass. Eur J Cardiothorac Surg 1998;14:101-3. 